\title{
FALTINGS' LOCAL-GLOBAL PRINCIPLE FOR THE FINITENESS OF LOCAL COHOMOLOGY MODULES
}

\author{
DAVOOD ASADOLLAHI AND REZA NAGHIPOUR*
}

\begin{abstract}
Let $(R, \mathfrak{m})$ be a complete local ring, $\mathfrak{a}$ an ideal of $R$ and $M$ a finitely generated $R$-module. The aim of this paper is to show that for any non-negative integer $n$, $f_{\mathfrak{a}}^{n}(M)=\inf \left\{0 \leq i \in \mathbb{Z} \mid \operatorname{dim} H_{\mathfrak{a}}^{i}(M) / N \geq n\right.$ for any finitely generated submodule $N \subseteq$ $\left.H_{\mathfrak{a}}^{i}(M)\right\}$, where $f_{\mathfrak{a}}^{n}(M):=\inf \left\{f_{\mathfrak{a} R_{\mathfrak{p}}}\left(M_{\mathfrak{p}}\right) \mid \mathfrak{p} \in \operatorname{Supp} M / \mathfrak{a} M\right.$ and $\left.\operatorname{dim} R / \mathfrak{p} \geq n\right\}$ is the $n$-th finiteness dimension of $M$ relative to $\mathfrak{a}$. As a consequence, it follows that the set

$$
\operatorname{Ass}_{R}\left(\oplus_{i=0}^{f_{\mathfrak{a}}^{n}(M)} H_{\mathfrak{a}}^{i}(M)\right) \cap\{\mathfrak{p} \in \operatorname{Spec} R \mid \operatorname{dim} R / \mathfrak{p} \geq n\}
$$

is finite. This generalizes the main result of Quy [10] and Brodmann-Lashgari [4].
\end{abstract}

\section{INTRODUCTION}

Throughout this paper, let $R$ denote a commutative Noetherian ring (with identity) and $\mathfrak{a}$ an ideal of $R$. For an $R$-module $M$, the $i^{\text {th }}$ local cohomology module of $M$ with support in $V(\mathfrak{a})$ is defined as:

$$
H_{\mathfrak{a}}^{i}(M)=\underset{n \geq 1}{\lim _{n \geq 1}} \operatorname{Ext}_{R}^{i}\left(R / \mathfrak{a}^{n}, M\right) .
$$

We refer the reader to [5] or [8] for more details about local cohomology. An important theorem in local cohomology is Faltings' Local-global Principle for the Finiteness Dimension of local cohomology modules [7, Satz 1], which states that for a positive integer $r$, the $R_{\mathfrak{p}}$-module $H_{\mathfrak{a} R_{\mathfrak{p}}}^{i}\left(M_{\mathfrak{p}}\right)$ is finitely generated for all $i \leq r$ and for all $\mathfrak{p} \in \operatorname{Spec} R$ if and only if the $R$-module $H_{\mathfrak{a}}^{i}(M)$ is finitely generated for all $i \leq r$.

Another formulation of Faltings' Local-global Principle, particularly relevant for this paper, is in terms of the generalization of the finiteness dimension $f_{\mathfrak{a}}(M)$ of $M$ relative to $\mathfrak{a}$, where

$$
f_{\mathfrak{a}}(M):=\inf \left\{i \in \mathbb{N}_{0} \mid H_{\mathfrak{a}}^{i}(M) \text { is not finitely generated }\right\},
$$

with the usual convention that the infimum of the empty set of integers is interpreted as $\infty$. It is well known that $f_{\mathfrak{a}}(M)=\inf \left\{f_{\mathfrak{a} R_{\mathfrak{p}}}\left(M_{\mathfrak{p}}\right) \mid \mathfrak{p} \in \operatorname{Supp} M / \mathfrak{a} M\right.$ and $\left.\operatorname{dim} R / \mathfrak{p} \geq 0\right\}$, see [5, 9.6.2]. Using this idea, for any non-negative integer $n, \mathrm{~K}$. Bahmanpour et al., in [3], introduced the notion of the $n$-th finiteness dimension $f_{\mathfrak{a}}^{n}(M)$ of $M$ relative to $\mathfrak{a}$ by

$$
f_{\mathfrak{a}}^{n}(M):=\inf \left\{f_{\mathfrak{a} R_{\mathfrak{p}}}\left(M_{\mathfrak{p}}\right) \mid \mathfrak{p} \in \operatorname{Supp} M / \mathfrak{a} M \text { and } \operatorname{dim} R / \mathfrak{p} \geq n\right\} .
$$

Key words and phrases. Associated primes, Faltings' local-global principle, Local cohomology.

2000 Mathematics Subject Classification: 13D45, 14B15, 13E05.

This research was in part supported by a grant from IPM.

*Corresponding author: e-mail: naghipour@ipm.ir (Reza Naghipour). 
Note that $f_{\mathfrak{a}}^{n}(M)$ is either a positive integer or $\infty$ and that $f_{\mathfrak{a}}^{0}(M)=f_{\mathfrak{a}}(M)$. Also, they showed that the least integer $i$ such that $H_{\mathfrak{a}}^{i}(M)$ is not minimax (resp. weakly Laskerian), equals to $f_{\mathfrak{a}}^{1}(M)$ (resp. $f_{\mathfrak{a}}^{2}(M)$ ). So it is rather natural to ask whether Faltings' Local-global Principle, as stated in $(\dagger)$, generalizes in the obvious way to the invariants $f_{\mathfrak{a}}^{n}(M)$. To this end, we are able to introduce the concept of an $R$-module in dimension $<n$ as a generalization of the notion of a FSF module [10. An $R$-module $M$ is said to be in dimension $<n$, if there is a finitely generated submodule $N$ of $M$ such that $\operatorname{dim} \operatorname{Supp} M / N<n$.

As a main result of this paper we shall show that:

Theorem 1.1. Let $(R, \mathfrak{m})$ be a complete local ring, $\mathfrak{a}$ an ideal of $R$ and $M$ a finitely generated $R$-module. Then for any $n \in \mathbb{N}_{0}$,

$$
f_{\mathfrak{a}}^{n}(M)=\inf \left\{0 \leq i \in \mathbb{Z} \mid H_{\mathfrak{a}}^{i}(M) \text { is not in dimension }<n\right\} .
$$

As a consequence of Theorem 1.2, we derive the following, which is a generalization of the main result of Quy [10, Theorem 3.2] and Brodmann-Lashgari [4, Theorem 2.2].

Corollary 1.2. Let $(R, \mathfrak{m})$ be a complete local ring, $\mathfrak{a}$ an ideal of $R$ and $M$ a finitely generated $R$-module. Then the set

is finite.

$$
\operatorname{Ass}_{R}\left(\bigoplus_{i=0}^{f_{\mathfrak{a}}^{n}(M)} H_{\mathfrak{a}}^{i}(M)\right) \cap\{\mathfrak{p} \in \operatorname{Spec} R \mid \operatorname{dim} R / \mathfrak{p} \geq n\}
$$

Throughout this paper, $R$ will always be a commutative Noetherian ring with non-zero identity and $\mathfrak{a}$ will be an ideal of $R$. By a skinny or weakly Laskerian module, we mean an $R$-module $M$ such that the set $\operatorname{Ass}_{R} M / N$ is finite, for each submodule $N$ of $M$ (cf. [11] or [6]). Moreover, an $R$-module $M$ is said to be minimax, if there exists a finitely generated submodule $N$ of $M$, such that $M / N$ is Artinian.

\section{The Main Results}

In [10], P. H. Quy introduced the class of FSF modules and he has given some properties and applications of this modules. An $R$-module $M$ is said to be a FSF module if there is a finitely generated submodule $N$ of $M$ such that support of the quotient module $M / N$ is finite. When $R$ is a Noetherian ring, it is clear that, if $M$ is FSF, then $\operatorname{dim} \operatorname{Supp} M / N \leq 1$. This motivates the definition.

Definition 2.1. Let $n$ be a non-negative integer. An $R$-module $M$ is said to be in dimension $<n$, if there is a finitely generated submodule $N$ of $M$ such that $\operatorname{dim} \operatorname{Supp} M / N<n$

Remark 2.2. Let $n$ be a non-negative integer and let $M$ be an $R$-module.

(i) If $n=0$, then $M$ is in dimension $<n$ if and only if $M$ is Noetherian.

(ii) If $M$ is minimax, then $M$ is in dimension $<1$. In particular, if $M$ is Noetherian or Artinian, then $M$ is in dimension $<1$.

(iii) If $M$ is FSF, then $M$ is in dimension $<2$.

(iv) If $M$ is skinny or weakly Laskerian, then $M$ is in dimension $<2$, by [1, Theorem 3.3]. 
(v) If $M$ is reflexive, then $M$ is in dimension $<1$.

(vi) If $M$ is linearly compact, then $M$ is in dimension $<1$. Recall that $M$ is said to be linearly compact if each system of congruences $x \equiv x_{i}\left(M_{i}\right)$ indexed by a set $I$, and where the $M_{i}$ are submodules of $M$, has a solution $x$ whenever it has a solution for every finite subsystem. It is know that it either $M$ reflexive or linearly compact, then $M$ is minimax (see e.g. [6]).

Definition 2.3. If $T$ is a subset of Spec $R$ and $n \in \mathbb{N}_{0}$, then we define

$$
(T)_{\geq n}=\{\mathfrak{p} \in T \mid \operatorname{dim} R / \mathfrak{p} \geq n\} .
$$

Definition 2.4. Let $n$ be a non-negative integer, $\mathfrak{a}$ an ideal of $R$ and $M$ an $R$-module. Then we define

$$
h_{\mathfrak{a}}^{n}(M)=\inf \left\{0 \leq i \in \mathbb{Z}: H_{\mathfrak{a}}^{i}(M) \text { is not in dimension }<n\right\} .
$$

Now we are prepared to state and prove the main result of this paper, which shows that the least integer $i$ such that $H_{\mathfrak{a}}^{i}(M)$ is not in dimension $<n$, equals to $\inf \left\{f_{\mathfrak{a} R_{\mathfrak{p}}}\left(M_{\mathfrak{p}}\right) \mid \mathfrak{p} \in\right.$ $\operatorname{Supp} M / \mathfrak{a} M$ and $\operatorname{dim} R / \mathfrak{p} \geq n\}$.

Theorem 2.5. Let $(R, \mathfrak{m})$ be a complete local ring, $\mathfrak{a}$ an ideal of $R$ and $M$ a finitely generated $R$-module. Then for any $n \in \mathbb{N}_{0}$,

$$
f_{\mathfrak{a}}^{n}(M)=h_{\mathfrak{a}}^{n}(M) .
$$

Proof. Let $i$ be a non-negative integer such that $H_{\mathfrak{a}}^{i}(M)$ is in dimension $<n$. Then it follows from the definition that there is a finitely generated submodule $N$ of $H_{\mathfrak{a}}^{i}(M)$ such that $\operatorname{dim} \operatorname{Supp} H_{\mathfrak{a}}^{i}(M) / N<n$. Thus for all $\mathfrak{p} \in \operatorname{Supp} M / \mathfrak{a} M$ with $\operatorname{dim} R / \mathfrak{p} \geq n$ we have $\left(H_{\mathfrak{a}}^{i}(M) / N\right)_{\mathfrak{p}}=0$. Therefore $\left(H_{\mathfrak{a}}^{i}(M)\right)_{\mathfrak{p}}$ is finitely generated and so $h_{\mathfrak{a}}^{n}(M) \leq f_{\mathfrak{a}}^{n}(M)$.

We now suppose that $t=h_{\mathfrak{a}}^{n}(M)<f_{\mathfrak{a}}^{n}(M)$, and look for a contradiction. To this end, first we show that for all $m \in \mathbb{N}$, the set $\left(\operatorname{Ass}_{R} H_{\mathfrak{a}}^{t}(M) /\left(0:_{H_{\mathfrak{a}}^{t}(M)} \mathfrak{a}^{m}\right)\right)_{\geq n}$ is finite. To achieve this, suppose the contrary is true. Then there exists a countably infinite subset $\left\{\mathfrak{p}_{k}\right\}_{k=1}^{\infty}$ of $\left(\operatorname{Ass}_{R} H_{\mathfrak{a}}^{t}(M) /\left(0:_{H_{\mathfrak{a}}^{t}(M)} \mathfrak{a}^{m}\right)\right)_{\geq n}$. Let $S$ be the multiplicatively closed subset $R \backslash \bigcup_{k=1}^{\infty} \mathfrak{p}_{k}$. We now show that the $S^{-1} R$-module $H_{S^{-1} \mathfrak{a}}^{t}\left(S^{-1} M\right)$ is finitely generated. To do this, in view of Faltings' Local-global Principle theorem (see [5, Theorem 9.6.1]), it is enough to show that for all $j \leq t$ and for all prime ideals $\mathfrak{p}$ with $S \cap \mathfrak{p}=\emptyset$, the $R_{\mathfrak{p}}$-module $\left(H_{S^{-1} \mathfrak{a}}^{j}\left(S^{-1} M\right)\right)_{S^{-1} \mathfrak{p}}$ is finitely generated. Since $S \cap \mathfrak{p}=\emptyset$, it follows that $\mathfrak{p} \subseteq \bigcup_{k=1}^{\infty} \mathfrak{p}_{k}$, and so by [9, Lemma 3.2] there exists $k \geq 1$ such that $\mathfrak{p} \subseteq \mathfrak{p}_{k}$. Thus $\operatorname{dim} R / \mathfrak{p} \geq n$. Now, as $j<f_{\mathfrak{a}}^{n}(M)$, it follows that $\left(H_{S^{-1} \mathfrak{a}}^{j}\left(S^{-1} M\right)\right)_{S^{-1} \mathfrak{p}} \cong\left(H_{\mathfrak{a}}^{j}(M)\right)_{\mathfrak{p}}$ is finitely generated, as required. Therefore the set $\operatorname{Ass}_{S^{-1} R} S^{-1}\left(H_{\mathfrak{a}}^{t}(M) /\left(0:_{H_{\mathfrak{a}}^{t}(M)} \mathfrak{a}^{m}\right)\right)$ is finite. On the other hand, we have $S^{-1} \mathfrak{p}_{k} \in \operatorname{Ass}_{S^{-1} R}\left(S^{-1} H_{\mathfrak{a}}^{t}(M) /\left(0:_{H_{\mathfrak{a}}^{t}(M)} \mathfrak{a}^{m}\right)\right)$ for all $k=1,2, \ldots$, which is a contradiction.

Consequently, for all $m \in \mathbb{N}$, the set $\left(\operatorname{Ass}_{R} H_{\mathfrak{a}}^{t}(M) /\left(0:_{H_{\mathfrak{a}}^{t}(M)} \mathfrak{a}^{m}\right)\right)_{\geq n}$ is finite. Now, we let $\mathbb{A}$ be the set of all prime ideals $\mathfrak{p}$ of $R$ such that there exists $m \in \mathbb{N}$ with

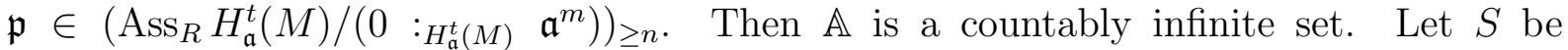
the multiplicatively closed subset $R \backslash \bigcup_{\mathfrak{p} \in \mathbb{A}} \mathfrak{p}$. Then it is easy to see that for all $m \in$ $\mathbb{N}$, the set $\operatorname{Ass}_{S^{-1} R} S^{-1}\left(H_{\mathfrak{a}}^{t}(M) /\left(0:_{H_{\mathfrak{a}}^{t}(M)} \mathfrak{a}^{m}\right)\right)$ is finite. Thus for all $m \in \mathbb{N}$, the set 
$\operatorname{Supp} S^{-1}\left(H_{\mathfrak{a}}^{t}(M) /\left(0:_{H_{\mathfrak{a}}^{t}(M)} \mathfrak{a}^{m}\right)\right)$ is a closed subset of Spec $R$ (in the Zariski topology), and so the descending chain

$$
\cdots \supseteq \operatorname{Supp} S^{-1}\left(H_{\mathfrak{a}}^{t}(M) /\left(0:_{H_{\mathfrak{a}}^{t}(M)} \mathfrak{a}^{m}\right)\right) \supseteq \operatorname{Supp} S^{-1}\left(H_{\mathfrak{a}}^{t}(M) /\left(0:_{H_{\mathfrak{a}}^{t}(M)} \mathfrak{a}^{m+1}\right)\right) \supseteq \cdots,
$$

is eventually stationary. Let $E_{m}$ denote its eventually stationary value, so that there is $m \in \mathbb{N}$ such that for all $l \geq m$,

$$
E_{m}=\operatorname{Supp} S^{-1}\left(H_{\mathfrak{a}}^{t}(M) /\left(0:_{H_{\mathfrak{a}}^{t}(M)} \mathfrak{a}^{l}\right)\right) .
$$

Since for all $i<t, H_{\mathfrak{a}}^{i}(M)$ is in dimension $<n$, it follows that $\left(0:_{H_{\mathfrak{a}}^{t}(M)} \mathfrak{a}^{m}\right)$ is also in dimension $<n$. Thus there is a finitely generated submodule $N$ of $\left(0:_{H_{\mathfrak{a}}^{t}(M)} \mathfrak{a}^{m}\right)$ such that $\operatorname{dim} \operatorname{Supp}\left(0:_{H_{\mathfrak{a}}^{t}(M)} \mathfrak{a}^{m}\right) / N<n$.

Now, we show that $\operatorname{dim} \operatorname{Supp} H_{\mathfrak{a}}^{t}(M) /\left(0:_{H_{\mathfrak{a}}^{t}(M)} \mathfrak{a}^{m}\right)<n$. Suppose the contrary is true. Then there exists $\mathfrak{q} \in \operatorname{Ass}_{R} H_{\mathfrak{a}}^{t}(M) /\left(0:_{H_{\mathfrak{a}}^{t}(M)} \mathfrak{a}^{m}\right)$ such that $\operatorname{dim} R / \mathfrak{q} \geq n$. Whence $\mathfrak{q} \in \mathbb{A}$, and so $S \cap \mathfrak{q}=\emptyset$. Thus $S^{-1} \mathfrak{q} \in E_{m}$. On the other hand, since $t<f_{\mathfrak{a}}^{n}(M)$ and $\operatorname{dim} R / \mathfrak{q} \geq n$, it yields that $\left(H_{\mathfrak{a}}^{t}(M)\right)_{\mathfrak{q}}$ is a finitely generated $R_{\mathfrak{q}}$-module. Therefore there exists $l \geq m$ such that $\left(\mathfrak{a} R_{\mathfrak{q}}\right)^{l}\left(H_{\mathfrak{a}}^{t}(M)\right)_{\mathfrak{q}}=0$, and so $\left(H_{\mathfrak{a}}^{t}(M) /\left(0:_{H_{\mathfrak{a}}^{t}(M)} \mathfrak{a}^{l}\right)\right)_{\mathfrak{q}}=0$. Hence we have $\left(S^{-1}\left(H_{\mathfrak{a}}^{t}(M) /\left(0:_{H_{\mathfrak{a}}^{t}(M)} \mathfrak{a}^{l}\right)\right)\right)_{S^{-1} \mathfrak{q}}=0$, and so $S^{-1} \mathfrak{q} \notin E_{m}$, which is a contradiction. Consequently, we have $\operatorname{dim} \operatorname{Supp} H_{\mathfrak{a}}^{t}(M) /\left(0:_{H_{\mathfrak{a}}^{t}(M)} \mathfrak{a}^{m}\right)<n$. Finally, from the exact sequence

$$
0 \longrightarrow\left(0:_{H_{\mathfrak{a}}^{t}(M)} \mathfrak{a}^{m}\right) / N \longrightarrow H_{\mathfrak{a}}^{t}(M) / N \longrightarrow H_{\mathfrak{a}}^{t}(M) /\left(0:_{H_{\mathfrak{a}}^{t}(M)} \mathfrak{a}^{m}\right) \longrightarrow 0,
$$

we conclude that $\operatorname{dim} \operatorname{Supp} H_{\mathfrak{a}}^{t}(M) / N<n$. That is the $R$-module $H_{\mathfrak{a}}^{t}(M)$ is in dimension $<n$, and so we have obtained a contradiction.

Corollary 2.6. Let $(R, \mathfrak{m})$ be a complete local ring, $\mathfrak{a}$ an ideal of $R$ and $M$ a finitely generated $R$-module. Then

(i) $f_{I}^{1}(M)=\inf \left\{i \in \mathbb{N}_{0} \mid H_{I}^{i}(M)\right.$ is not minimax $\}$.

(ii) $f_{I}^{2}(M)=\inf \left\{i \in \mathbb{N}_{0} \mid H_{I}^{i}(M)\right.$ is not weakly Laskerian $\}$.

Proof. The result follows from the definition and Theorem 2.5.

Corollary 2.7. Let $(R, \mathfrak{m})$ be a complete local ring, $\mathfrak{a}$ an ideal of $R$ and $M$ a finitely generated $R$-module. Then the set $\left(\operatorname{Ass}_{R}\left(\oplus_{i=0}^{t-1} H_{\mathfrak{a}}^{i}(M)\right)\right)_{\geq n}$ is finite, where $t=f_{\mathfrak{a}}^{n}(M)$.

Proof. In view of Theorem 2.5, the $R$-module $\oplus_{i=0}^{t-1} H_{\mathfrak{a}}^{i}(M)$ is in dimension $<n$. So there is a finitely generated submodule $N$ of $\oplus_{i=0}^{t-1} H_{\mathfrak{a}}^{i}(M)$ such that $\operatorname{dim} \oplus_{i=0}^{t-1} H_{\mathfrak{a}}^{i}(M) / N \leq n-1$. Now the assertion follows immediately from the exact sequence

$$
0 \longrightarrow N \longrightarrow \oplus_{i=0}^{t-1} H_{\mathfrak{a}}^{i}(M) \longrightarrow \oplus_{i=0}^{t-1} H_{\mathfrak{a}}^{i}(M) / N \longrightarrow 0 .
$$

The final result is a generalization of the main result of Quy [10, Theorem 3.2] and Brodmann-Lashgari [4, Theorem 2.2] for complete local rings.

Theorem 2.8. Let $(R, \mathfrak{m})$ be a complete local ring, $\mathfrak{a}$ an ideal of $R$ and $M$ a finitely generated $R$-module. Then the set $\left(\operatorname{Ass}_{R}\left(\oplus_{i=0}^{t} H_{\mathfrak{a}}^{i}(M)\right)\right)_{\geq n}$ is finite, where $t=f_{\mathfrak{a}}^{n}(M)$. 
Proof. Using Corollary 2.7 it is enough to show that the set $\left(\operatorname{Ass}_{R} H_{\mathfrak{a}}^{t}(M)\right)_{\geq n}$ is finite. To this end, suppose that the contrary is true. Then there exists a countably infinite subset $\left\{\mathfrak{q}_{k}\right\}_{k=1}^{\infty}$ of $\left(\operatorname{Ass}_{R} H_{\mathfrak{a}}^{t}(M)\right)_{\geq n}$, and so by [9, Lemma 3.2], $\mathfrak{p} \nsubseteq \bigcup_{k=1}^{\infty} \mathfrak{q}_{k}$, for every $\mathfrak{p} \in$ Spec $R$ with $\operatorname{dim} R / \mathfrak{p}<n$. Let $S$ be the multiplicatively closed subset $R \backslash \bigcup_{k=1}^{\infty} \mathfrak{q}_{k}$. Then, it easily follows from [5, Theorem 9.6.1] that $S^{-1} H_{\mathfrak{a}}^{i}(M)$ is a finitely generated $S^{-1} R$-module, for all $i=0,1, \ldots, t-1$ and so in view of [2, Theorem 2.3], the $R$-module

$$
\operatorname{Hom}_{S^{-1} R}\left(S^{-1} R / S^{-1} \mathfrak{a}, S^{-1} H_{\mathfrak{a}}^{t}(M)\right),
$$

is finitely generated. Therefore the set

$$
\operatorname{Ass}_{S^{-1} R} S^{-1} H_{\mathfrak{a}}^{t}(M)
$$

is finite. But $S^{-1} \mathfrak{q}_{k} \in \operatorname{Ass}_{S^{-1} R} S^{-1}\left(H_{\mathfrak{a}}^{i}(M)\right)$ for all $k=1,2, \ldots$, which is a contradiction.

\section{Acknowledgments}

The authors would like to thank Professors Hossein Zakeri and Kamal Bahmanpour for reading of the first draft and valuable discussions. Also, we would like to thank from the Institute for Research in Fundamental Sciences (IPM), for its financial support.

\section{REFERENCES}

[1] K. Bahmanpour, On the equivalence of FSF and weakly Laskerian modules, Math. Scand. to appear.

[2] K. Bahmanpour and R. Naghipour, On the cofiniteness of local cohomology modules, Proc. Amer. Math. Soc. 136(2008), 2359-2363.

[3] K. Bahmanpour, R. Naghipour and M. Sedghi, Minimaxness and cofiniteness properties of local cohomology modules, Comm. Algebra, to appear.

[4] M.P. Brodmann and F.A. Lashgari, A finiteness result for associated primes of local cohomology modules, Proc. Amer. Math. Soc. 128(2000), 2851-2853.

[5] M.P. Brodmann and R.Y. Sharp, Local cohomology; an algebraic introduction with geometric applications, Cambridge University Press, Cambridge, 1998.

[6] K. Divaani-Aazar and A. Mafi, Associated primes of local cohomology modules, Proc. Amer. Math. Soc. 133(2005), 655-660.

[7] G. Faltings, Der endlichkeitssatz in der lokalen kohomologie, Math. Ann. 255(1981), 45-56.

[8] A. Grothendieck, Local cohomology, Notes by R. Hartshorne, Lecture Notes in Math., 862 (Springer, New York, 1966).

[9] T. Marley and J.C. Vassilev, Cofiniteness and associated primes of local cohomology modules, J. Algebra 256(2002), 180-193.

[10] P. H. Quy, On the finiteness of associated primes of local cohomology modules, Proc. Amer. Math. Soc. 138 (2010), 1965-1968.

[11] H. Robbins, Associated primes of local cohomology modules and $S_{2}$-ification, J. Pure and Appl. Algebra 216(2012), 519-523.

Department of Mathematics, University of Tabriz, Tabriz, Iran; and School of Mathematics, Institute for studies in Theoretical Phisics and Mathematics (IPM), P.O. Box 19395-5746, TEHran, Iran.

E-mail address: naghipour@ipm.ir

E-mail address: naghipour@tabrizu.ac.ir

E-mail address: d_asadollahi@tabrizu.ac.ir 